\title{
FunPred 3.0: Improved protein function prediction using protein interaction network
}

\author{
Sovan Saha ${ }^{1}$, Piyali Chatterjee ${ }^{2}$, Subhadip Basu ${ }^{3}$, Mita Nasipuri ${ }^{3}$, Dariusz Plewczynski ${ }^{\text {Corresp. } 4,5}$ \\ ${ }^{1}$ Department of Computer Science and Engineering, Dr. Sudhir Chandra Sur Degree Engineering College, Kolkata, West Bengal, India \\ 2 Department of Computer Science and Engineering, Netaji Subhash Engineering College, Kolkata, India \\ 3 Department of Computer Science and Engineering, Jadavpur University, Kolkata, West Bengal, India \\ 4 Laboratory of Functional and Structural Genomics, Centre of New Technologies, University of Warsaw, Warsaw, Poland \\ 5 Faculty of Mathematics and Information Science, Warsaw University of Technology, Warsaw, Poland \\ Corresponding Author: Dariusz Plewczynski \\ Email address: d.plewczynski@cent.uw.edu.pl
}

Proteins are the most versatile macromolecules in living systems and perform crucial biological functions. In the advent of the post genomic era, the next generation sequencing is done routinely at the population scale for a variety of species. The challenging problem is to massively determine the functions of proteins that are yet not characterized by detailed experimental studies. Identification of protein functions experimentally is a laborious and time-consuming task involving many resources. We therefore propose the automated protein function prediction methodology using in silico algorithms trained on carefully curated experimental datasets. We present the improved protein function prediction tool FunPred 3.0, an extended version of our previous methodology FunPred 2, which exploits neighbourhood properties in protein-protein interaction network (PPIN) and physicochemical properties of amino acids. Our method is validated using the available functional annotations in the PPIN network of Saccharomyces cerevisiae in the latest Munich Information Center for Protein (MIPS) dataset. The PPIN data of Saccharomyces cerevisiae in MIPS dataset includes 4554 unique proteins in 13528 protein-protein interactions (PPINs) after the elimination of the self-replicating and the selfinteracting protein pairs. Using the developed FunPred 3.0 tool, we are able to achieve the mean precision, the recall and the F-score values of $0.55,0.82$ and 0.66 respectively. FunPred 3.0 is then used to predict the functions of unpredicted protein pairs (incomplete and missing functional annotations) in MIPS dataset of Saccharomyces cerevisiae. The method is also capable of predicting the subcellular localization of proteins along with its corresponding functions. The code and the complete prediction results are available freely at: https://github.com/SovanSaha/FunPred-3.0.git. 


\section{FunPred 3.0: Improved protein function prediction using 2 protein interaction network}

3 Sovan Saha ${ }^{1}$, Piyali Chatterjee ${ }^{2}$, Subhadip Basu ${ }^{3}$, Mita Nasipuri ${ }^{3}$, Dariusz Plewczynski ${ }^{4,5}$

$4{ }^{1}$ Department of Computer Science and Engineering, Dr. Sudhir Chandra Sur Degree Engineering 5 College, DumDum, Kolkata, India

$6 \quad{ }^{2}$ Department of Computer Science and Engineering, Netaji Subhash Engineering College, Garia, Kolkata, 7 India

$8{ }^{3}$ Department of Computer Science and Engineering, Jadavpur University, Kolkata, West Bengal, India

$9{ }^{4}$ Laboratory of Functional and Structural Genomics, Centre of New Technologies, University of Warsaw, 10 Warsaw, Poland

$11{ }^{5}$ Faculty of Mathematics and Information Science, Warsaw University of Technology, Warsaw, Poland

12

13 Corresponding Author:

14 Dariusz Plewczynski ${ }^{4,5}$

15 Banacha 2c Street, 02-097 Warsaw, Poland

16 Email address: d.plewczynski@cent.uw.edu.pl 


\section{ABSTRACT}

Proteins are the most versatile macromolecules in living systems and perform crucial biological functions. In the advent of the post genomic era, the next generation sequencing is done routinely at the population scale for a variety of species. The challenging problem is to massively determine the functions of proteins that are yet not characterized by detailed experimental studies. Identification of protein functions experimentally is a laborious and time-consuming task involving many resources. We therefore propose the automated protein function prediction methodology using in silico algorithms trained on carefully curated experimental datasets. We present the improved protein function prediction tool FunPred 3.0, an extended version of our previous methodology FunPred 2, which exploits neighbourhood properties in protein-protein interaction network (PPIN) and physicochemical properties of amino acids. Our method is validated using the available functional annotations in the PPIN network of Saccharomyces cerevisiae in the latest Munich Information Center for Protein (MIPS) dataset. The PPIN data of Saccharomyces cerevisiae in MIPS dataset includes 4554 unique proteins in 13528 proteinprotein interactions (PPINs) after the elimination of the self-replicating and the self-interacting protein pairs. Using the developed FunPred 3.0 tool, we are able to achieve the mean precision, the recall and the F-score values of $0.55,0.82$ and 0.66 respectively. FunPred 3.0 is then used to predict the functions of unpredicted protein pairs (incomplete and missing functional annotations) in MIPS dataset of Saccharomyces cerevisiae. The method is also capable of predicting the subcellular localization of proteins along with its corresponding functions. The code and the complete prediction results are available freely at: https://github.com/SovanSaha/FunPred-3.0.git.

\section{INTRODUCTION}

Proteins with similar functions are more likely to interact. If the function of one protein is known then the functions of the binding un-annotated protein may either be experimentally assigned or computationally predicted (Chatterjee et al. 2011a; Chatterjee et al. 2011b; Moosavi et al. 2013; Prasad et al. 2017; Saha et al. 2012; Saha et al. 2014; Sriwastava et al. 2015). Several computational techniques have been developed using either the protein sequence ( $\mathrm{Ng} \&$ Henikoff 2003), protein structure (Lee et al. 2007; Mills et al. 2015), protein-protein interactions (Moosavi et al. 2013; Schwikowski et al. 2000; Vazquez et al. 2003; Xiong et al. 2013), or sequence motifs or signatures (Chatterjee et al. 2011a; Chen et al. 2007; Lichtarge et al. 1996). Protein Interaction datasets are represented as graphs (with every node corresponding to an individual protein and each edge between a pair of nodes representing the interaction between them) can be used to assign biological functions to a protein with an assumption that close neighbors of a protein are functionally similar. 
Protein function prediction problem is characterized by several factors like the diversity of members for functional groups, the hierarchical relationships among functional classes, incomplete or missing information about proteins and their functions. Thus, it defines a complex multi-label learning problem (Jiang \& McQuay 2012; Valentini 2014; Zhang \& Zhou 2014). Hierarchical relationships among labels are described in MIPS Functional Catalogue and Gene Ontology. Valentini (Valentini 2014) uses a binary classifier for each label according to True Path Rule (TPR) and the funCat. Recent work of Guoxian and the co-authors (Yu et al. 2015), explored the incomplete label problem in a hierarchical manner using function correlation. Another approach for predicting protein function, as proposed by Piovesan et al. (Piovesan et al. 2015) includes the combination of the trio: PPIN information, protein domain and sequence. In another work, Zhao et al. (Zhao et al. 2016) invokes dynamic weighted interaction network instead of the static one. This dynamic network is enriched with PPIN, time course gene expression data, protein's domain information and protein complex information which ultimately predict function of a protein using majority ranking. While most of the predictive models highlights on the most highly related similar proteins in the neighborhood of the test protein, Reinders et al. (Reinders et al. 2018) focuses on the less similar proteins. It is shown by the application of Label-Space Dimensionality Reduction (LSDR) techniques that though these proteins are less similar but they are quite informative and plays an important role in protein function prediction. Another iterative algorithm is implemented by Sun et al. (Sun et al. 2018) for predicting protein functions. It is completely dependent on the identification of the functional dependencies which are based on proteins and their interactions. Sequence similarity network is another important aspect for protein function prediction, which is considered in the development of the methodology, Effusion, as proposed in the work of Yunes \& Babbitt (Yunes \& Babbitt 2018). Other notable works in this field are Wang et al. (Wang et al. 2018) and Fa et al. (Fa et al. 2018)

All these methods discussed above have already taken protein function prediction to the next higher level. Yet, the still uncovered details in the study and analysis support the need for new computational methods exploiting the protein-protein interaction networks for biological function identification. In our novel methodology FunPred 3.0, the functions of test proteins are determined by analyzing the neighbourhood properties of their protein interaction network. At the same time, certain selected physicochemical properties of amino acids are also used along with it. This task is challenging because of several reasons; e.g., large number of functional groups, different levels of the interconnection hierarchy, proteins with multiple functional groups, and incomplete or missing labels. In this proposed methodology, MIPS dataset (Mewes et al. 2002) is used. It contains protein pairs along with their corresponding functions. At the initial phase, to estimate the effectiveness of FunPred 3.0, essential proteins are selected as test proteins. The functions of these proteins are considered to be unknown for experimental purpose though their functions are defined in the dataset. Then we have applied FunPred 3.0 to predict the functions of the test proteins. Predicted functions are hence matched with the original ones to compute precision, recall and F-Score. While executing FunPred 3.0, it has been observed that 870 PPIs out of 13528 PPIs i.e., $\sim 6.4 \%$ of the overall MIPS dataset (Mewes et al. 2002) are unpredicted i.e. either unknown or missing. FunPred 3.0 has been also applied to predict the unannotated protein function and protein interaction (Mamoon et al. 2010), and also assigning the functional annotations for 767 PPIs out of 870 PPIs, representing circa $\sim 5.7 \%$ of the overall MIPS dataset. Similar instances have been also observed in the case of subcellular location of proteins where 1679 proteins out of 6721 unique number of proteins are still unpredicted i.e. the 
subcellular localization of these proteins are still unknown. The predicted functional annotations and subcellular localization of these unpredicted proteins and protein pairs respectively result in relevant biological information, such as vital processes, diseases related mutations.

\section{METHODOLOGY}

In one of our two earlier works, Funpred-1 (Saha et al. 2014), the selection of ten percent of test proteins of the top eight functional groups from the dataset was done randomly. Top eight functional groups were selected on the basis of maximum number of occurrences and interactions of proteins in them. While in another, FunPred 2 (Saha et al. 2017a), protein clusters are formed initially by the application of node and edge weight. Then fifty percent of proteins from each of the formed clusters are selected as test proteins. In both the cases, test proteins are chosen randomly. Since both these works are completely based on protein function prediction from PPIN, so network formed for each test protein is extensively large (up to level - 2) enough to process which gradually enhances the overall computational overhead. So randomness is basically implemented to filter out the most essential proteins out of the entire PPIN and select them as the test protein. But, variation of test set (i.e. proteins beyond ten percent in FunPred-1 or fifty percent in FunPred 2) as well as application of node and edge weight thresholds (in FunPred 2) might also play an important role in prediction accuracy level which has not been yet tested so far. It may be also considered as a major drawback and limitation of our previous two methodologies, which has been tried to overcome in FunPred 3.0. FunPred 3.0 is an extended and advanced version of FunPred 2. Though the basic outlay of the both is same but the uniqueness of FunPred 3.0 can be defined in a three way approach:

1. Application of three levels of threshold for the formation of clusters and selecting all proteins from the clusters as test set to overcome the limitations of its predecessors.

2. Incorporation of feature selection.

3. Capable of prediction of functions of unpredicted protein pairs (incomplete and missing functional annotations) in MIPS dataset of Saccharomyces cerevisiae.

4. Capable of prediction of subcellular localization of proteins.

PPIN formed of proteins and their corresponding interactions may contain essential/nonessential proteins as well as reliable/unreliable edges. Proteins having maximum number of interconnected neighbors are considered as essential proteins while proteins having less number of interconnected neighbors are considered as non-essential ones. Presence of non-essential neighbors in the PPIN might affect the unknown protein function prediction level accuracy. So proper identification and elimination of non-essential proteins is needed to ensure the presence of maximum number of essential proteins in the PPIN. In the proposed work, detection of essential proteins is implemented by node weight. Node weight (Wang \& Wu 2013) basically assigns a weightage score to each node or protein based on its corresponding degree. High node weight determines essential while low node weight detects non-essential protein. Thus non-essential proteins are discarded from PPIN along their corresponding edges. Even after this initial phase of PPIN refinement, there are still some unreliable edges present in the network. Since protein clusters are formed, so it is obvious that two nodes with an edge between them belong to the same cluster if they have high similarity. Edge between two nodes of high similarity is 
147

considered as reliable edge while that of low similarity is denoted as unreliable edge. In the proposed work, detection of reliable edges is executed by edge weight. Edge weight (Wang \& $\mathrm{Wu}$ 2013) also assigns a weightage score to each edge connected by two proteins in the terminals. Assignment of edge weight to an edge depends on the number of common neighbors between the two terminal proteins of the corresponding edge. More number of common neighbors signifies high similarity which in turn detects reliable edges. On the other hand, unreliable edges have low similarity since they have less number of common neighbors. Thus, unreliable edges are identified and pruned from the PPIN. Filtered out PPIN after these refinements, contains only essential proteins and reliable edges, which ultimately helps in enhancing prediction accuracy level.

In newly proposed algorithm, FunPred 3.0, first detects protein cluster and then selects all the proteins as test proteins from different predicted clusters. We have adopted the approach of forming protein cluster as mentioned in the work of Wang and Wu (Wang \& Wu 2013). Protein clusters, thus formed, comprises of proteins belonging to any functional group. It results in accumulating larger number of functional groups as compared with only 8 functional groups in our previous work (Saha et al. 2014). The novel computational method works in two stages:

1. All the unique proteins are first clustered into $M$ mutually exclusive clusters based on their node weight and edge weight in the overall PPIN. Node and edge weight have been used to ensure all the most essential nodes with higher reliability are present in the cluster and get selected as test proteins.

2. Functional annotations are then derived from the multi-level neighbourhood of an unknown protein within each cluster.

More specifically, FunPred 3.0 is categorized into two sections: FunPred 3.0_Clust and FunPred 3.0_Pred.

FunPred 3.0_Clust uses the node weight and edge weight properties to rank and cluster all the proteins, creating M mutually exclusive protein clusters (Wang \& Wu 2013). The number of functional labels, associated with each interacting pair is large and in some cases annotations in each such cluster is unpredicted (incomplete or missing). This fact forces us to heuristically choose the node and edge weight threshold values, such that the unlabelled proteins are associated with larger protein clusters and have many neighbourhood interactions (see Figure 1 and Figure 2). Three thresholds (high, medium and low) are set for each of node and edge weight using equation 1 (Zhang et al. 2016).

$$
T h_{k}=\alpha+k \cdot \sigma \cdot\left(1-\frac{1}{1+\sigma^{2}}\right)
$$

where for node weight/edge weight, $\mathrm{k} \in\{1,2,3\}$ denotes three different thresholds i.e. low, medium and high respectively. $\alpha$ is the mean of node weight/edge weight values of all proteins. $\sigma$ is the standard deviation of node weight/edge weight values of all proteins. Proteins and edges having value less value than these node and edge weight thresholds get discarded and are considered as non-essential proteins and unreliable edges in the network respectively.

The entire methodology has been described in Algorithm 1 as well as pictorially highlighted in Figure 1 and Figure 2. In Figure 1, sample Table 1 (node weight table) is formed 
188

189

190

191

192

193

194

195

196

197

198

199

200

201

202

203

204

205

206

207

208

209

210

211

212

213

214

215

216

217

218

219

220

221

222

223

224

225

226

227

228

229

230

231

from the initial PPIN of yeast. Hence, node weight threshold is calculated using equation 1 at three levels: high, medium and low. These thresholds are applied on the initial PPIN to filter out three sub-networks i.e. sub-network1, sub-network2, sub-network3 respectively at high, medium and low node weight thresholds. Respective edge weight tables i.e. sample Table 2 (in Figure 1), sample Table 3 (in Figure 1), sample Table 4 (in Figure 1) are formed from sub-network1, subnetwork2, sub-network3, upon which high, medium and low edge weight thresholds (obtained using equation 1) are applied to form pruned sub-network4, sub-network5 and sub-network3.

In Figure 2, sample Table 5 (node weight table formed from sample Table 1 under high threshold in Figure 1) has been generated from refined sub-network4 which afterwards is sorted in descending order to form sample Table 6 (in Figure 2). From sample Table 6 (in Figure 2), first protein having the highest node weight is selected as seed of the initial cluster. Then corresponding level 1 neighbors of the seed are included in the cluster provided its inclusion in the cluster does not let the edge weight to fall below the high threshold (verified using sample Table 2 in Figure 1). The entire initial cluster contents are discarded from sample Table 6 (in Figure 2) and the next cluster is formed with the next selected seed. This process continues until all the proteins in sample Table 6 (in Figure 2) get clustered. Thus M mutually exclusive protein clusters are formed where $1=<M<=N 4$ (N4 is the total number of proteins present in sample Table 6 in Figure 2). The entire procedure is repeated for refined sub-network5 and refined subnetwork6 until M mutually exclusive protein clusters is formed for each of them.

All the proteins belonging to M mutually exclusive protein clusters (under three levels of thresholds: high, medium and low separately), obtained from FunPred 3.0_Clust are considered as essential proteins and hence they are included in the test set of our proposed methodology. In the second step, FunPred 3.0_Pred predicts labels these selected unlabelled (or test) proteins using neighbourhood properties and physicochemical properties of amino acids (see Algorithm 2 and Figure 3). In Figure 3, for each test protein (say $P_{1}$ belonging to refined sub-network4 in Figure 1) its level 1 neighborhood graph is formed including those proteins which are present in its corresponding node weight table: sample Table 1 under high threshold in Figure 1. Then protein clusters are formed at level 1 (considering each level 1 protein as the seed of the cluster) in a similar way as formed in FunPred 3.0_Clust. It should be noted here that the number of clusters formed here is equivalent to the number of proteins in level 1. Distance between the mean of the physico-chemical features of each protein cluster as well as test protein is computed and the test belongs to the cluster having the least distance. All the functions of the selected cluster are allocated to the test protein.

The relevant level -1 neighbours of the test proteins are chosen to form their individual neighbourhood graph. In finding the level-1 neighbours or forming their individual neighbourhood graph, relevance is measured in terms of edge weight properties. Next, PCP score is computed for the neighbourhood graph of each test protein. Six different high-ranked physico-chemical features: aliphatic index (Singh et al. 2008), gravy (Kyte \& Doolittle 1982), aromacity (Lobry \& Gautier 1994), number of negatively charged residues (Singh et al. 2008), number of positively charged residues (Singh et al. 2008), isoelectric point (Bjellqvist et al. 1994) are used to reckon this physico-chemical property (PCP) based score. These high-ranked features are selected from ten divergent physico-chemical features (see supplementary) by the enactment of four distinct classifiers: XGBoost classifier (Chen \& Guestrin 2016; Pedregosa et al. 2011), Random Forest classifier (Breiman 2001; Pedregosa et al. 2011), Extra Tree classifier

Peer) reviewing PDF | (2018:07:29585:3:0:CHECK 8 Mar 2019) 
232 (Geurts et al. 2006; Pedregosa et al. 2011) and Recursive feature elimination classifier 233 (Pedregosa et al. 2011). High-ranked five among ten features have been picked at first by each 234 classifier. Then from these picked features, frequency of maximum occurrences for each 235 individual feature has been noted from which endmost six features get selected (see Table 1). 236 Finally, each test protein is assigned to a functional group of the neighbourhood graph, based on 237 the nearest neighbourhood approach on the basis of mean PCP score. FunPred 3.0_Clust (see 238 Algorithm 1) and FunPred 3.0_Pred (see Algorithm 2) describes the methodology of unknown 239 protein selection and function prediction respectively. 
Algorithm 1. FunPred 3.0_Clust:

(For formation of protein clusters which consist of essential proteins and reliable edges)

Input: $\quad$ Undirected PPIN $G$.

Output: Protein clusters at three levels of threshold: high, medium and low

Begin

//computation of node weight of $G$

for all nodes in $G$

compute node weight

//computation of node weight threshold

compute node weight threshold at three levels: high, medium and low using

equation 1

//Elimination of non-essential proteins based on node weight threshold

for each level of threshold

for all nodes in $G$

if node weight does not exceed threshold remove corresponding node.

//Formation of refined sub-networks $\mathrm{G}_{\text {high }}{ }^{\prime}, \mathrm{G}_{\text {medium }}{ }^{\prime}$ and $\mathrm{G}_{\mathrm{low}}{ }^{\prime}$ from $G$

$\mathrm{G}_{\text {high }}{ }^{\prime}, \mathrm{G}_{\text {medium }}$ ' and $\mathrm{G}_{\text {low }}{ }^{\prime}$ consisting of only essential proteins (high node weight) are

formed

//computation of edge weight of $G^{\prime}$

for all edges in $G_{\text {high }}$, $G_{\text {medium }}$ and $G_{\text {low }}{ }^{\prime}$

compute edge weight

//computation of edge weight threshold

compute edge weight threshold at three levels: high, medium and low using equation 1

//Elimination of unreliable edges based on edge weight threshold

for all edges in $\mathrm{G}_{\text {high }}$

if edge weight does not exceed high level of edge threshold

remove corresponding edge.

repeat the same for low, medium level of threshold and $G_{\text {medium }}$ and $G_{\text {low }}{ }^{\prime}$ respectively.

//Formation of refined sub-networks $\mathrm{G}_{\text {high,high }}, \mathrm{G}_{\text {medium,medium }}$ and $\mathrm{G}_{\text {low,low }}$ from $\mathrm{G}_{\text {high }}{ }^{\prime}, \mathrm{G}_{\text {medium }}$ ' and $\mathrm{G}_{\text {low }}$ at high node and edge weight threshold, medium node and edge weight threshold, low node and edge weight threshold respectively.

form $G_{\text {high,high }}, G_{\text {medium,medium }}$ and $\mathrm{G}_{\text {low,low }}$ consisting of only reliable edges (high edge weight)

//Formation of clusters at three levels of thresholds

for all proteins in $\mathrm{G}_{\text {high,high }}$

form node weight table

sort the node weight table based on the node weights

select the first protein $P$ in the node weight table as the seed of initial cluster $C_{M}^{\text {high }}$

i.e. $C_{M}^{\text {high }}=\{P\}$ where $1=<\mathrm{M}<=\mathrm{W}$ ( $\mathrm{W}$ is the total no. of nodes in node weight table)

neighbors of $P$ are added to $C_{M}^{\text {high }}$ provided its inclusion does not cause edge weight to fall below high edge weight threshold value i.e. $C_{M}^{\text {high }}=\{P\} \cup N_{P_{1}}$

update the node edge table by eliminating all the proteins present in $C_{M}^{h i g h}$ and continue with the next seed to form clusters in the same way mentioned above till all the proteins in the node weight table belongs to a cluster.

repeat the same procedure for $G_{\text {medium,medium }}$ and $G_{\text {low,low }}$. 
Algorithm 2. FunPred 3.0_Pred:

(Protein function prediction of test proteins)

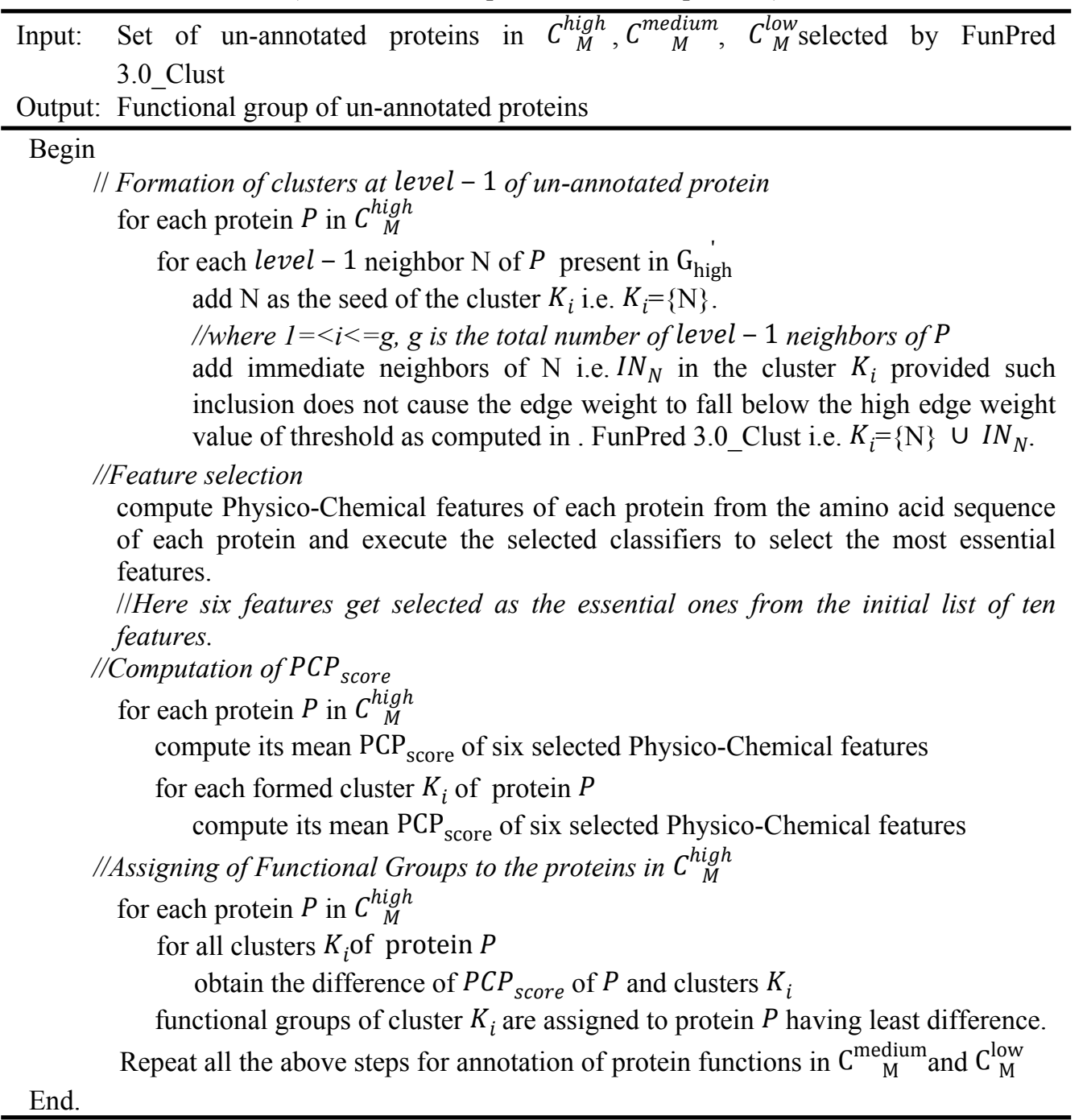


It needs to be highlighted here that both FunPred 3.0 Clust and FunPred 3.0 Pred have been executed at three levels: 1. High node and edge weight threshold, 2. Medium node and edge weight threshold, 3. Low node and edge weight threshold. So, we have tested FunPred 3.0 at each of the three levels to assess its performance impact.

Besides, predicting protein function, it has been observed that the protein subcellular localization is yet another important aspect which needs to be considered since it helps in better understanding of protein function. So, subcellular localization dataset of yeast has been obtained from UniProt database (Apweiler et al. 2004). On careful observation it has been noted that there are 6721 unique number of proteins out of which localization of some proteins are still unpredicted. It is similar as that of $6.4 \%$ of the overall MIPS dataset (Mewes et al. 2002) which are unpredicted i.e. either unknown or missing. So FunPred 3.0 is also implemented to predict these unpredicted protein subcellular localization.

In FunPred 3.0, the subcellular localization dataset of yeast is centrally categorized under three major sections: proteins residing in nucleus (termed as nuclear proteins), proteins residing in cytoplasm (termed as cytoplasm proteins) and proteins residing in other regions (termed as interface proteins). Besides these, there is also another section termed as unpredicted localization proteins, consisting of those whose localization are not yet predicted (see Figure 4, Figure 5 and Figure 6). So before dealing with the unpredicted localization proteins, the predictive accuracy of FunPred 3.0 needs to be assessed just in a similar way earlier as that of protein function prediction. Same test set of essential proteins (as generated by FunPred 3.0_Clust) of yeast (MIPS) is also considered here (localization of which are known but considered to be unknown for experimental purpose). Selected test and candidate proteins in the PPIN of nuclear, cytoplasm and interface proteins are highlighted in Figure 7, Figure 8 and Figure 9 respectively. Now for each test protein, its corresponding level -1 neighborhood graph is formed. In a PPIN, a protein almost shares similar properties as that of its neighborhood proteins. The same is also applicable to the test protein but all the properties or functions of the neighborhood cannot be transmitted to it. So proper assessment needs to be implemented in the neighborhood of the test protein. For this purpose, FunPred 3.0_Pred_SL (SL stands for Subcellular Localization) is applied. It first assigns respective subcellular localization information to the neighborhood of the test protein using UniProt database. Hence it counts the frequency of occurrence of each subcellular location (nucleus, cytoplasm or any other region). The subcellular location having the highest frequency of occurrence among the neighborhood is allocated to the test protein. The test protein becomes nuclear or cytoplasm or interface proteins according to the allocated subcellular location. Then the allocated subcellular localization is checked from the Uniprot database. The overall result which is achieved by the application of FunPred 3.0_Pred_SL, has been highlighted in Table 2. An overall accuracy of $69.1 \%, 57 \%$ and $53 \%$ is reckoned for nuclear, cytoplasm and interface proteins respectively. It is observed from Table 2, that our method fails to predict the subcellular localization of few proteins among all the three categories. This is because our method mainly predicts subcellular localization using PPIN neighborhood based approaches and if there is practically no information or significantly less amount of interactive information in the PPIN for a particular test protein then our method fails. This can be considered as one of our limitations which can be redressed in future works by incorporating protein sequence.

Subcellular localization dataset of yeast contains 1679 number of unpredicted localization proteins out of which our method predicts the localization of 638 proteins successfully. 
285

Localization information for the remaining 1041 proteins cannot be predicted because of the absence of PPIN interaction in MIPS dataset as discussed earlier. This extra added layer of biological information about subcellular localization of proteins along with the protein function prediction boost up our methodology FunPred 3.0 to the next higher level.

\section{RESULTS}

Initially, PPIN of yeast consists of 4554 unique proteins in 13528 protein-protein interactions (PPINs) after the elimination of the self-replicating and the self-interacting protein pairs. After the network refinement through the execution of node and edge weight threshold, non-essential proteins along with unreliable edges get eliminated and the initial PPIN gets reduced to almost 3174 unique proteins and 6936 PPINs (approx.) considering three levels of thresholds from which FunPred 3.0_Clust form protein clusters to generate test set of proteins.

During the result analysis it is observed that proteins belonging to random functional groups like lipid metabolism, DNA Repair etc. get selected as test proteins. In FunPred 3.0_Pred, all proteins from each protein cluster formed from FunPred 3.0_Clust are considered as test proteins. The overall initial PPIN of yeast is highlighted in Figure 10 while in Figure 11, 433 test proteins (most essential ones among the refined PPIN of yeast consisting of 3174 unique proteins and 6936 PPINs) selected by FunPred 3.0_Clust at high node and edge weight threshold values, are highlighted in yellow circle (shape) in the initial PPIN of yeast consisting of 4554 unique proteins in 13528 protein-protein interactions (PPINs). It should be noted in Figure 11, that all the selected test proteins belongs to the most densely connected region of PPIN which establishes the fact that these are indeed most strongly connected essential proteins. The performance of FunPred 3.0 is evaluated using standard performance measures, such as Precision $(\mathrm{P})$, Recall $(\mathrm{R})$ and F-Score $(\mathrm{F})$ values, which are calculated using the following equations:

$$
\mathrm{P}=\frac{T P}{T P+F P} \quad \mathrm{R}=\frac{T P}{T P+F N} \quad \mathrm{~F}=\frac{2 *(P * R)}{P+R}
$$

where TP, FP, FN represent True Positive, False Positive and False Negative respectively.

The performance of FunPred 3.0 has been analyzed under different levels of thresholds of node and edge weights as highlighted in Table 3. It should be noted here that under high and medium thresholds, same Precision, Recall and F-score have been retrieved since number of selected test proteins are equivalent in both the cases. All over result analysis as depicted in Table 3 shows that there are not much significant changes in the result as such varying the thresholds except a slight fall in precision and F-score under low threshold as compared to the others. The fall is due to the relaxation in the node and edge weight thresholds resulting in incorporation of less essential proteins in the test set. So, the high threshold ensures inclusion of the most essential proteins. So the Precision, Recall and F-score of FunPred 3.0 are reckoned as $0.55,0.82$ and 0.66 respectively under high threshold. High Recall and low Precision emerges out as a major characteristic of FunPred 3.0 when compared to the other methodologies except FunPred-2. It highlights the fact that most of the admissible results are successfully generated by FunPred 3.0. Table 4 shows a detailed performance comparison of other methodologies along with our proposed systems (like FunPred 1.1, FunPred 1.2 (Saha et al. 2014)), neighbourhood counting method (Schwikowski et al. 2000), the 
chi-square method (Hishigaki et al. 2001), a recent version of the neighbor relativity coefficient (NRC) (Moosavi et al. 2013), FPred_Apriori (Prasad et al. 2017), Zhang methodology (Zhang et al. 2009), Domain Combination Similarity (DCS) (Peng et al. 2014), Domain Combination Similarity in context of protein complexes (DSCP) (Peng et al. 2014), Protein Overlap Network (PON) (Liang et al. 2013), Deep_GO (Kulmanov et al. 2018) and the FS-weight based method (Chua et al. 2006)). All these data are collected from their respective works which are executed on the same organism i.e. yeast. The results of Deep_GO (Kulmanov et al. 2018) are computed manually for yeast dataset, the code of which is available at https://github.com/SovanSaha/FunPred-3.0.git. From Table 4, it can be also highlighted that our method, FunPred 3.0, yields relatively higher F-Score values than the others including its earlier version FunPred-2.

Table 4 also discloses the fact that NRC method has overpowered the rest except FunPred 1.1 (Saha et al. 2014), FunPred 1.2 (Saha et al. 2014), FPred_Apriori (Prasad et al. 2017) and FunPred 3.0. The reason behind this is observed as follows: Both version of FunPred 1 has incorporated two levels (i.e. level-1 and level-2) of PPIN as well as lot of essential neighborhood properties like neighborhood ratio, protein path connectivity and relative functional similarity (includes both ancestor and descendant information of a specific protein) have been utilized to assess the reliability of each node (protein) along with its associated edges (protein interaction) during the unannotated protein function prediction. FPred_Apriori (Prasad et al. 2017) executes both closeness centrality and edge clustering coefficient to make its predictive approach more effective than the others. Last but not least, FunPred 3.0 combines physico-chemical property of each protein along with neighborhood analysis (like node weight, edge weight etc.) for predicting protein function which ultimately promotes it to the next higher level in the terms of performance analysis when compared to the others.

Though Neighborhood counting method is simple in nature yet the performance measure of it has descended considerably in comparison to NRC (Moosavi et al. 2013), FS-weight \#1 (directly connected proteins) and FS-weight \#1 and \#2 (directly and indirectly connected proteins) despite of its simplicity (Chua et al. 2006). This is because no differentiation has been observed between the direct and indirect neighborhood connection. Beside most of the methods included in Table 4 like NRC (Moosavi et al. 2013), Chi square \#1\&2 (Hishigaki et al. 2001), Chi square \#1 (Hishigaki et al. 2001), Neighborhood counting \#1\&2 (Schwikowski et al. 2000), Neighborhood counting \#1 (Schwikowski et al. 2000) etc. are not utilized for the refinement of the PIN by pruning unreliable proteins or edges which in turn increases false positives in their prediction accuracy level. In FPred_Apriori (Prasad et al. 2017), a bottom-up predictor of existing Apriori algorithm has been utilized for protein function prediction by exploiting two most important neighborhood properties: closeness centrality and edge clustering coefficient of protein interaction network. Though the method is unique in the fact that the functions of the leaf nodes in the interaction network have been back propagated and thus labeled up to the root node (test protein) but yet it fails to generate high Recall and F-score than FunPred 3.0. But it returns substantially high precision values than the others as well as all our methods. DCS (Peng et al. 2014), DSCP (Peng et al. 2014), PON (Liang et al. 2013), Deep_GO (Kulmanov et al. 2018) and Zhang methodology (Zhang et al. 2009) are well developed methods for protein function prediction incorporating domain specific as well as neighborhood based properties but they fail to compete with all our methodologies due to the lack of important feature selection methodologies of physicochemical properties and proper assessment of nodes and edges involved in test set protein function prediction through node and edge weights. 
During experimental evaluation, the validation set is prepared with 4554 labeled Saccharomyces cerevisiae proteins, collected from the MIPS dataset. Using FunPred 3.0_Clust, we identify M mutually exclusive protein clusters (Wang \& Wu 2013). Experimental variations with $\mathrm{k}=1,2,3$, are included in Table 2 . Using an optimal choice of $\mathrm{k}=3$, we identify 433 test targets for the validation set. Now, the functional labels of these test proteins are assigned using FunPred 3.0_Pred. The Precision, Recall, F-scores of our method over the test targets of the validation set is obtained as $0.55,0.82$ and 0.66 respectively.

\section{DISCUSSION}

Our results (characterized by the Precision, Recall and F-Score) and comparison with the other protein functional group prediction models show the superiority of our approach. The FunPred 3.0 software has better performance than any existing function prediction in silico method. The network structure may be pruned based on the edge weight and along with it use of physicochemical properties lead to improved and faster functional prediction in complex and diverse protein-protein interaction networks. We would like to estimate the effectiveness of our in silico method for other organisms, such as in human protein-protein interactions with even more complex network architectures.

The initial results motivate us to predict the subcellular localization and unpredicted protein pair functions (missing/unknown functions) for 870 PPIs extracted from MIPS dataset. A protein can perform multiple functions in isolation. It may also perform some specific functions while interacting with one protein while perform some other specific functions while reacting with other proteins. But considering the fact that a protein often shares similar functions with proteins that interact with it (Chakicherla et al. 2018; Chatterjee et al. 2012; Shatsky et al. 2016), each protein pair is disintegrated in two constituent proteins and functions of each protein is predicted using FunPred 3.0. For an unknown protein pair $\mathrm{P}_{1} \mathrm{P}_{2}$, we predict the functions as an intersection of FunPred 3.0_Pred $\left(\mathrm{P}_{1}\right) \cap$ FunPred 3.0_Pred $\left(\mathrm{P}_{2}\right)$. The results of all the predicted annotations for the MIPS dataset are available at https:/github.com/SovanSaha/FunPred-3.0.git. Examples of the prediction of protein function and interactions for unpredicted pairs (both unknown and missing protein pair) have been shown in Table 5 and Table 6 respectively.

Summarizing, 767 unpredicted protein pair functions (511 unknown protein pair functions and 256 missing protein pair functions) in the MIPS dataset could be predicted using our FunPred 3.0 algorithm. Our approach failed to predict 103 unpredicted protein pairs since they have less number of acceptable neighbors. Simultaneously our methodology also performs very well in predicting subcellular localization of proteins as discussed earlier in the methodology section earlier. All the datasets and supplementary files are also freely available at https://github.com/SovanSaha/FunPred-3.0.git.

\section{CONCLUSION}

FunPred 3.0 thus proved to be an improved and advanced version of our previous 
408

409

410

411

412

413

414

415

416

417

418

419

420

421

422

423

424

425

426

427

428

429

430

431

432

433

434

435

436

437

438

439

440

441

442

443

444

445

446

447

448

449

450

451

452

453

454

455

methodology FunPred-2. The enhanced performance of FunPred 3.0 is due to the use of node weight, edge weight, and physicochemical properties of proteins in the prediction pathway of test set of proteins. It should be highlighted here that FunPred 3.0 incorporates the most essential features classified through four classifiers: XGBoost, Random Forest, Extra Tree and Recursive feature elimination. Recursive feature elimination (RFE) which indeed plays an important role in improving the performance of the proposed methodology. Though this method does not consider dynamic PPIN and integration of other multiple types of data like domain (Chatterjee et al. 2011a; Chatterjee et al. 2011b) etc., but topological analysis, association between function and protein have been proven to be significant for this research. Besides use of FunPred 3.0 to detect the subcellular localization of proteins as well as function of unpredicted protein pair functions (unknown and missing pairs of proteins) in MIPS database add an extra dimension to this work. Incorporation of other protein related features and their integration, use of the other benchmark datasets for different organism may give a proper insight of prediction. Beside this unannotated protein function prediction, the methodology behind the FunPred 3.0 algorithm can be also used in disease specific datasets (Saha et al. 2017b) also which may be a future direction as well. In a nutshell, the work presented here proposes the statistical learning evaluation of various features for prediction of protein functions in the complex yeast PPIN with reasonable accuracy. The dataset used in this study and the complete source codes of the FunPred 3.0 software package are available in the public domain (https://github.com/SovanSaha/FunPred-3.0.git) for non- commercial research.

\section{REFERENCES}

Apweiler R, Bairoch A, Wu CH, Barker WC, Boeckmann B, Ferro S, Gasteiger E, Huang H, Lopez R, Magrane M, Martin MJ, Natale DA, O'Donovan C, Redaschi N, and Yeh L-SL. 2004. UniProt: the Universal Protein knowledgebase. Nucleic Acids Research 32(Database issue):D115-D119. 10.1093/nar/gkh131.

Bjellqvist B, Basse B, Olsen E, and Celis JE. 1994. Reference points for comparisons of two-dimensional maps of proteins from different human cell types defined in a $\mathrm{pH}$ scale where isoelectric points correlate with polypeptide compositions. ELECTROPHORESIS 15(1):529-539. 10.1002/elps.1150150171.

Breiman L. 2001. Random Forests. Machine Learning 45(1):5-32. 10.1023/a:1010933404324.

Chakicherla A, Ligon Dang M, Rodriguez V, Hansen J, and Zemla A. 2018. Function prediction of an interacting protein pair using domain fusion analysis: SpaR and SpaK.

Chatterjee P, Basu S, Kundu M, Nasipuri M, and Plewczynski D. 2011a. PPI_SVM: Prediction of protein-protein interactions using machine learning, domain-domain affinities and frequency tables. Cellular \& Molecular Biology Letters 16(2):264-278. 10.2478/s11658-011-0008-x.

Chatterjee P, Basu S, Kundu M, Nasipuri M, and Plewczynski D. 2011b. PSP_MCSVM: brainstorming consensus prediction of protein secondary structures using two-stage multiclass support vector machines. Journal of Molecular Modeling 17(9):2191. 10.1007/s00894-011-1102-8.

Chatterjee T, Chatterjee P, Basu S, Kundu M, and Nasipuri M. 2012. Protein function by minimum distance classifier from protein interaction network. In: 2012 International Conference on Communications, Devices and Intelligent Systems (CODIS).588-591.

Chen J, Hsu W, Lee ML, and Ng SK. 2007. Labeling network motifs in protein interactomes for protein function prediction. In: 2007 IEEE 23rd International Conference on Data Engineering.546-555.

Peer) reviewing PDF | (2018:07:29585:3:0:CHECK 8 Mar 2019) 
456

457

458

459

460

461

462

463

464

465

466

467

468

469

470

471

472

473

474

475

476

477

478

479

480

481

482

483

484

485

486

487

488

489

490

491

492

493

494

495

496

497

498

499

500

501

502

503

504

505

506

507

508

509

510

Chen T, and Guestrin C. 2016. XGBoost: A Scalable Tree Boosting System. In: Proceedings of the 22nd ACM SIGKDD International Conference on Knowledge Discovery and Data Mining. San Francisco, California, USA: ACM.785-794.

Chua HN, Sung W-K, and Wong L. 2006. Exploiting indirect neighbours and topological weight to predict protein function from protein-protein interactions. Bioinformatics 22(13):1623-1630. 10.1093/bioinformatics/btl145.

Fa R, Cozzetto D, Wan C, and Jones DT. 2018. Predicting human protein function with multi-task deep neural networks. PLOS ONE 13(6):e0198216. 10.1371/journal.pone.0198216.

Geurts P, Ernst D, and Wehenkel L. 2006. Extremely randomized trees. Machine Learning 63(1):3-42. 10.1007/s10994-006-6226-1.

Hishigaki H, Nakai K, Ono T, Tanigami A, and Takagi T. 2001. Assessment of prediction accuracy of protein function from protein-protein interaction data. Yeast 18(6):523-531. 10.1002/yea.706.

Jiang JQ, and McQuay LJ. 2012. Predicting Protein Function by Multi-Label Correlated Semi-Supervised Learning. IEEE/ACM Transactions on Computational Biology and Bioinformatics 9(4):1059-1069. 10.1109/TCBB.2011.156.

Kulmanov M, Khan MA, Hoehndorf R, and Wren J. 2018. DeepGO: predicting protein functions from sequence and interactions using a deep ontology-aware classifier. Bioinformatics (Oxford, England) 34(4):660-668. 10.1093/bioinformatics/btx624.

Kyte J, and Doolittle RF. 1982. A simple method for displaying the hydropathic character of a protein. Journal of Molecular Biology 157(1):105-132. https://doi.org/10.1016/0022-2836(82)90515-0.

Lee D, Redfern O, and Orengo C. 2007. Predicting protein function from sequence and structure. Nature Reviews Molecular Cell Biology 8(995. 10.1038/nrm2281

Liang S, Zheng D, Standley DM, Guo H, and Zhang C. 2013. A novel function prediction approach using protein overlap networks. BMC Systems Biology 7(1):61. 10.1186/1752-0509-7-61.

Lichtarge O, Bourne HR, and Cohen FE. 1996. An Evolutionary Trace Method Defines Binding Surfaces Common to Protein Families. Journal of Molecular Biology 257(2):342-358. https://doi.org/10.1006/jmbi.1996.0167.

Lobry JR, and Gautier C. 1994. Hydrophobicity, expressivity and aromaticity are the major trends of amino-acid usage in 999 Escherichia coli chromosome-encoded genes. Nucleic Acids Research 22(15):3174-3180.

Mamoon R, Sumathy R, and Gajendra PSR. 2010. A Simple Approach for Predicting Protein-Protein Interactions. Current Protein \& Peptide Science 11(7):589-600. http://dx.doi.org/10.2174/138920310794109120.

Mewes HW, Frishman D, Güldener U, Mannhaupt G, Mayer K, Mokrejs M, Morgenstern B, Münsterkötter M, Rudd S, and Weil B. 2002. MIPS: a database for genomes and protein sequences. Nucleic Acids Research 30(1):31-34.

Mills CL, Beuning PJ, and Ondrechen MJ. 2015. Biochemical functional predictions for protein structures of unknown or uncertain function. Computational and Structural Biotechnology Journal 13(182-191. https://doi.org/10.1016/j.csbj.2015.02.003.

Moosavi S, Rahgozar M, and Rahimi A. 2013. Protein function prediction using neighbor relativity in proteinprotein interaction network. Computational Biology and Chemistry 43(11-16. https://doi.org/10.1016/j.compbiolchem.2012.12.003.

Peer) reviewing PDF | (2018:07:29585:3:0:CHECK 8 Mar 2019) 
511

512

513

514

515

516

517

518

519

520

521

522

523

524

525

526

527

528

529

530

531

532

533

534

535

536

537

538

539

540

541

542

543

544

545

546

547

548

549

550

551

552

553

554

555

556

557

558

559

560

561

562

563

564
Ng PC, and Henikoff S. 2003. SIFT: predicting amino acid changes that affect protein function. Nucleic Acids Research 31(13):3812-3814.

Pedregosa F, Varoquaux G, Gramfort A, Michel V, Thirion B, Grisel O, Blondel M, Prettenhofer P, Weiss R, Dubourg V, Vanderplas J, Passos A, Cournapeau D, Brucher M, Perrot M, and Duchesnay E. 2011. Scikitlearn: Machine Learning in Python. J Mach Learn Res 12(2825-2830.

Peng W, Wang J, Cai J, Chen L, Li M, and Wu F-X. 2014. Improving protein function prediction using domain and protein complexes in PPI networks. BMC Systems Biology 8(1):35. 10.1186/1752-0509-8-35.

Piovesan D, Giollo M, Leonardi E, Ferrari C, and Tosatto SCE. 2015. INGA: protein function prediction combining interaction networks, domain assignments and sequence similarity. Nucleic Acids Research 43(Web Server issue):W134-W140. 10.1093/nar/gkv523.

Prasad A, Saha S, Chatterjee P, Basu S, and Nasipuri M. 2017. Protein Function Prediction from Protein Interaction Network Using Bottom-up L2L Apriori Algorithm. In: Computational Intelligence, Communications, and Business Analytics. Singapore: Springer Singapore.3-16.

Reinders MJT, van Ham RCHJ, and Makrodimitris S. 2018. Improving protein function prediction using protein sequence and GO-term similarities. 10.1093/bioinformatics/bty751.

Saha S, Chatterjee P, Basu S, Kundu M, and Nasipuri M. 2012. Improving prediction of protein function from protein interaction network using intelligent neighborhood approach. In: 2012 International Conference on Communications, Devices and Intelligent Systems (CODIS).584-587.

Saha S, Chatterjee P, Basu S, Kundu M, and Nasipuri M. 2014. FunPred-1: Protein function prediction from a protein interaction network using neighborhood analysis. Cellular \& Molecular Biology Letters 19(4):675691. 10.2478/s11658-014-0221-5.

Saha S, Chatterjee P, Basu S, and Nasipuri M. 2017a. Functional Group Prediction of Un-annotated Protein by Exploiting Its Neighborhood Analysis in Saccharomyces Cerevisiae Protein Interaction Network. In: Chaki R, Saeed K, Cortesi A, and Chaki N, eds. Advanced Computing and Systems for Security: Volume Four. Singapore: Springer Singapore, 165-177.

Saha S, Sengupta K, Chatterjee P, Basu S, and Nasipuri M. 2017b. Analysis of protein targets in pathogen-host interaction in infectious diseases: a case study on Plasmodium falciparum and Homo sapiens interaction network. Briefings in Functional Genomics:elx024-elx024. 10.1093/bfgp/elx024.

Schwikowski B, Uetz P, and Fields S. 2000. A network of protein-protein interactions in yeast. Nature Biotechnology 18(1257. 10.1038/82360

Shatsky M, Allen S, Gold BL, Liu NL, Juba TR, Reveco SA, Elias DA, Prathapam R, He J, Yang W, Szakal ED, Liu H, Singer ME, Geller JT, Lam BR, Saini A, Trotter VV, Hall SC, Fisher SJ, Brenner SE, Chhabra SR, Hazen TC, Wall JD, Witkowska HE, Biggin MD, Chandonia J-M, and Butland G. 2016. Bacterial Interactomes: Interacting Protein Partners Share Similar Function and Are Validated in Independent Assays More Frequently Than Previously Reported. Molecular \& Cellular Proteomics : MCP 15(5):1539-1555. 10.1074/mcp.M115.054692.

Singh M, Wadhwa PK, and Kaur S. 2008. Predicting Protein Function using Decision Tree. World Academy of Science, Engineering and Technology 2(3):300-303.

Sriwastava BK, Basu S, and Maulik U. 2015. Predicting Protein-Protein Interaction Sites with a Novel Membership Based Fuzzy SVM Classifier. IEEE/ACM Transactions on Computational Biology and Bioinformatics 12(6):1394-1404. 10.1109/TCBB.2015.2401018.

Peer] reviewing PDF | (2018:07:29585:3:0:CHECK 8 Mar 2019) 
565

566

567

568

569

570

571

572

573

574

575

576

577

578

579

580

581

582

583

584

585

586

587

588

589

590

591

592

593

594

595

596

597

598

599

600

601

602

Sun P, Tan X, Guo S, Zhang J, Sun B, Du N, Wang H, and Sun H. 2018. Protein Function Prediction Using Function Associations in Protein-Protein Interaction Network. IEEE Access 6(30892-30902. 10.1109/ACCESS.2018.2806478.

Valentini G. 2014. Hierarchical Ensemble Methods for Protein Function Prediction. ISRN Bioinformatics 2014(34. $10.1155 / 2014 / 901419$.

Vazquez A, Flammini A, Maritan A, and Vespignani A. 2003. Global protein function prediction from proteinprotein interaction networks. Nature Biotechnology 21(697. 10.1038/nbt825

Wang S, and Wu F. 2013. Detecting overlapping protein complexes in PPI networks based on robustness. Proteome Science 11(Suppl 1):S18-S18. 10.1186/1477-5956-11-S1-S18.

Wang Z, Zhao C, Wang Y, Sun Z, and Wang N. 2018. PANDA: Protein function prediction using domain architecture and affinity propagation. Scientific Reports 8(1):3484. 10.1038/s41598-018-21849-1.

Xiong W, Liu H, Guan J, and Zhou S. 2013. Protein function prediction by collective classification with explicit and implicit edges in protein-protein interaction networks. BMC Bioinformatics 14(Suppl 12):S4-S4. 10.1186/1471-2105-14-S12-S4.

Yu G, Zhu H, and Domeniconi C. 2015. Predicting protein functions using incomplete hierarchical labels. $B M C$ Bioinformatics 16(1. 10.1186/s12859-014-0430-y.

Yunes JM, and Babbitt PC. 2018. Effusion: prediction of protein function from sequence similarity networks. Bioinformatics 35(3):442-451. 10.1093/bioinformatics/bty672.

Zhang ML, and Zhou ZH. 2014. A Review on Multi-Label Learning Algorithms. IEEE Transactions on Knowledge and Data Engineering 26(8):1819-1837. 10.1109/TKDE.2013.39.

Zhang S, Chen H, Liu K, and Sun Z. 2009. Inferring protein function by domain context similarities in proteinprotein interaction networks. BMC Bioinformatics 10(1):395. 10.1186/1471-2105-10-395.

Zhang Y, Lin H, Yang Z, Wang J, Liu Y, and Sang S. 2016. A method for predicting protein complex in dynamic PPI networks. BMC Bioinformatics 17(7):229. 10.1186/s12859-016-1101-y.

Zhao B, Wang J, Li M, Li X, Li Y, Wu FX, and Pan Y. 2016. A New Method for Predicting Protein Functions From Dynamic Weighted Interactome Networks. IEEE Transactions on NanoBioscience 15(2):131-139. 10.1109/TNB.2016.2536161.

PeerJ reviewing PDF | (2018:07:29585:3:0:CHECK 8 Mar 2019) 
Figure 1

\section{Application of Node Weight and Edge Weight at three levels of threshold: High, Medium and Low in FunPred 3.0_Clust}

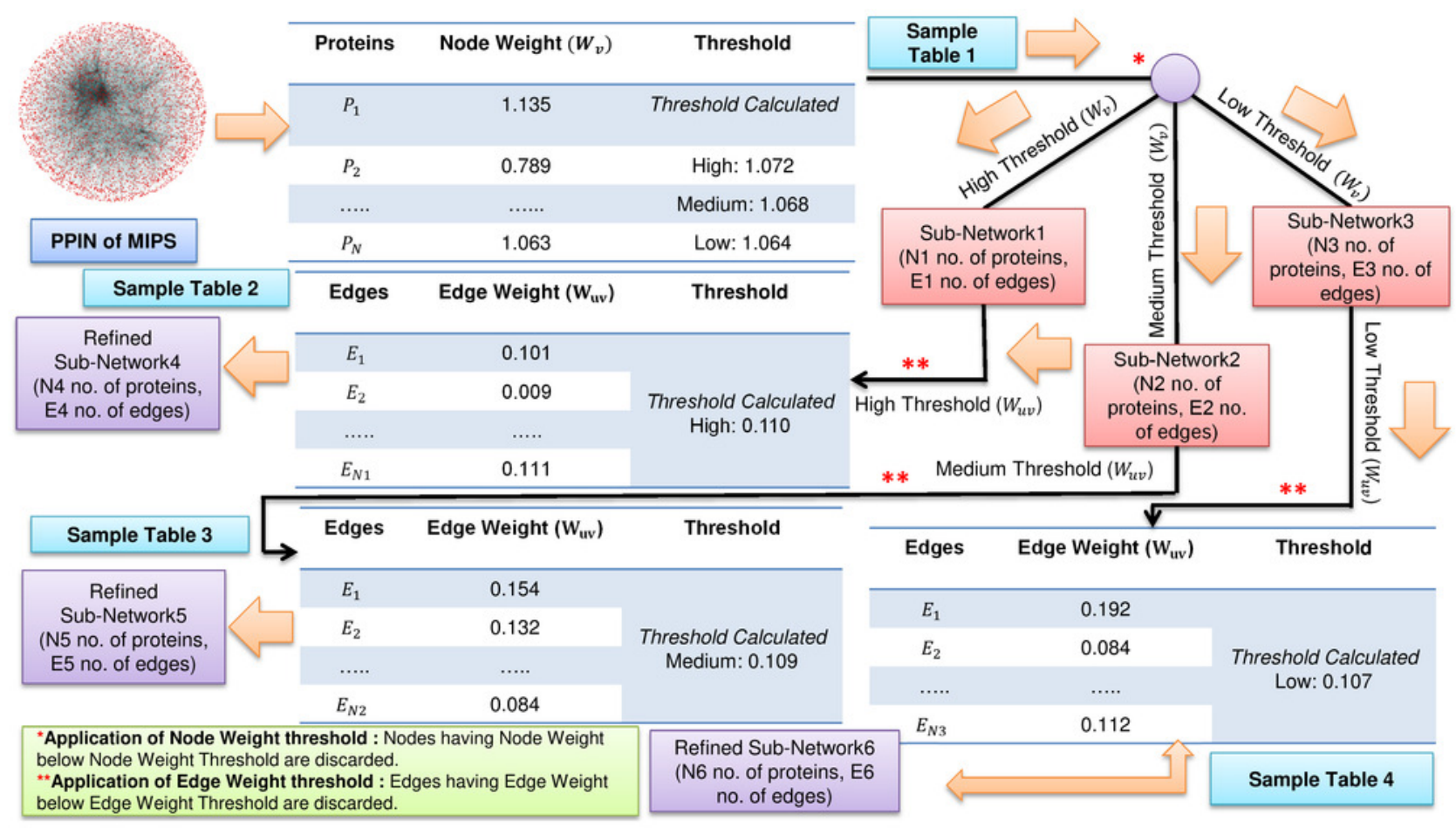


Figure 2

\section{Formation of clusters from refined network after application of three levels of Node and Edge Weight threshold in FunPred 3.0_Clust}

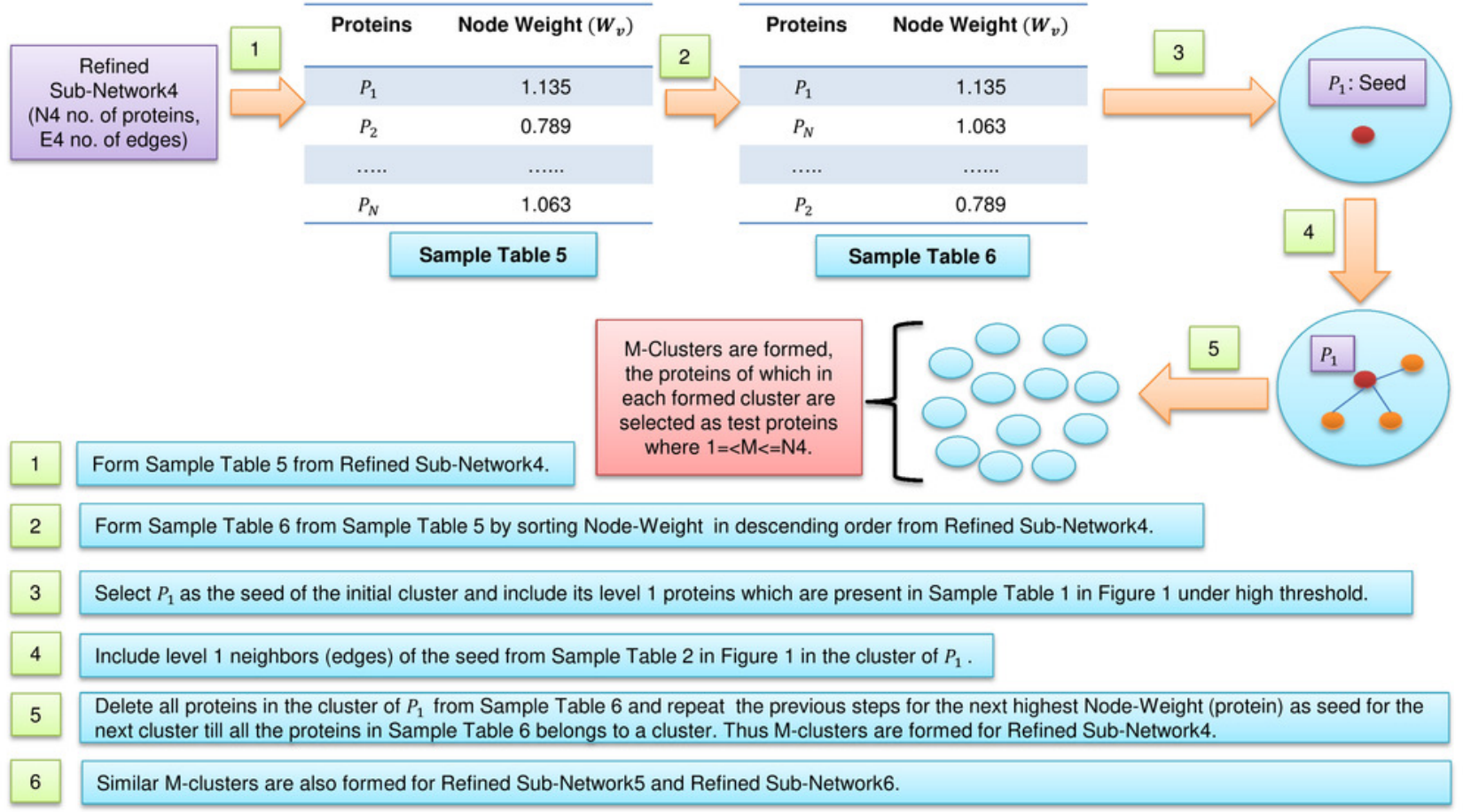


Figure 3

\section{Working Model of FunPred 3.0_Pred}
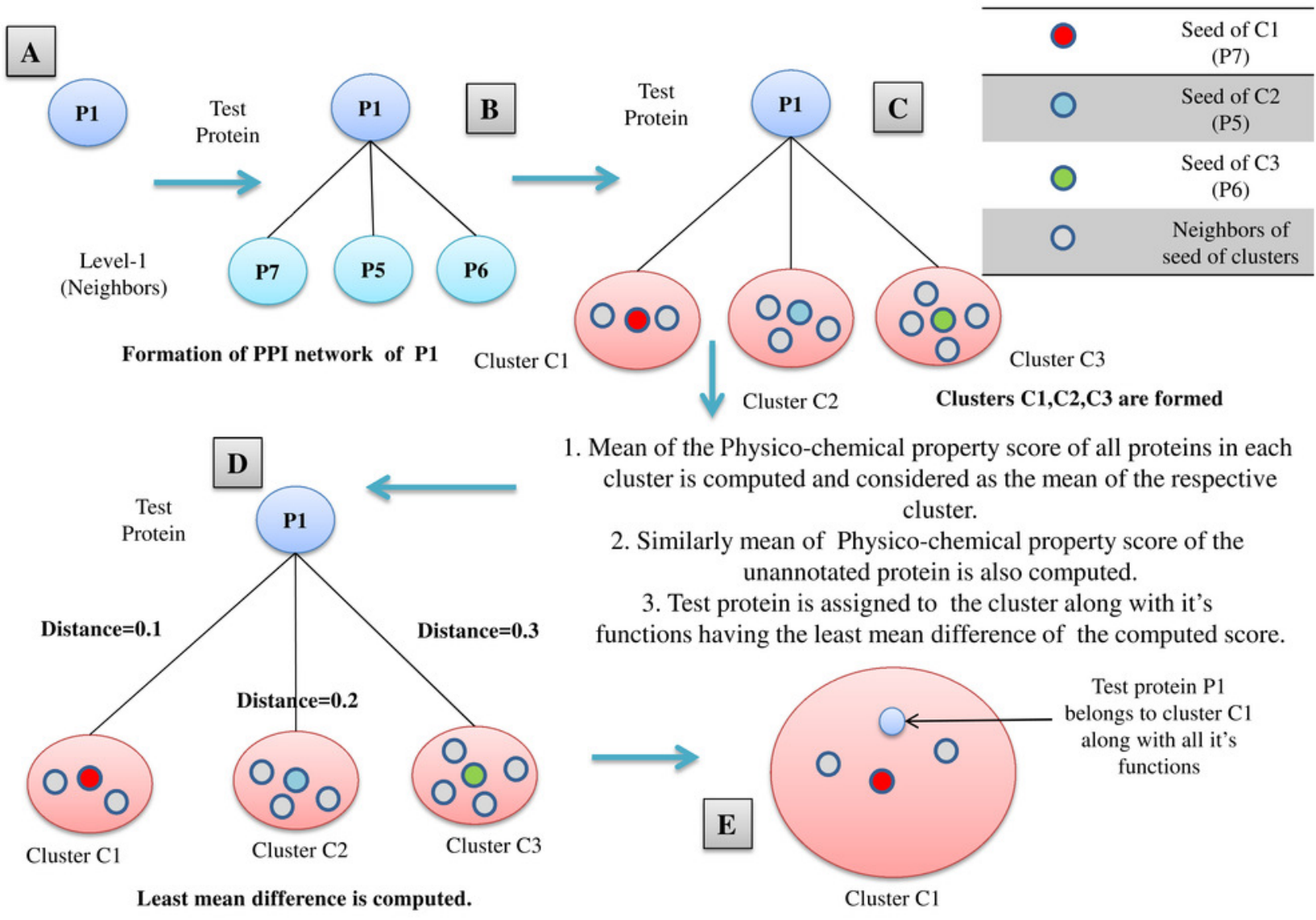

1. Mean of the Physico-chemical property score of all proteins in each cluster is computed and considered as the mean of the respective cluster.

2. Similarly mean of Physico-chemical property score of the unannotated protein is also computed.

3. Test protein is assigned to the cluster along with it's functions having the least mean difference of the computed score.

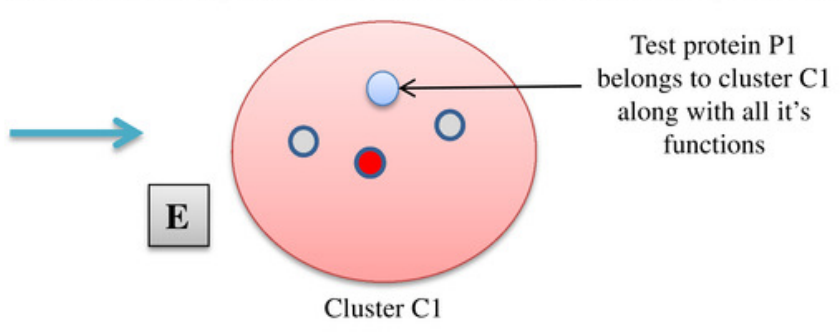

Predicted cluster for test protein 
Figure 4

PPIN of Yeast (Saccharomyces cerevisiae): Cytoplasm proteins (red), Nuclear Proteins (Green), Interface Proteins (Blue), Unpredicted localization Proteins (Orange)

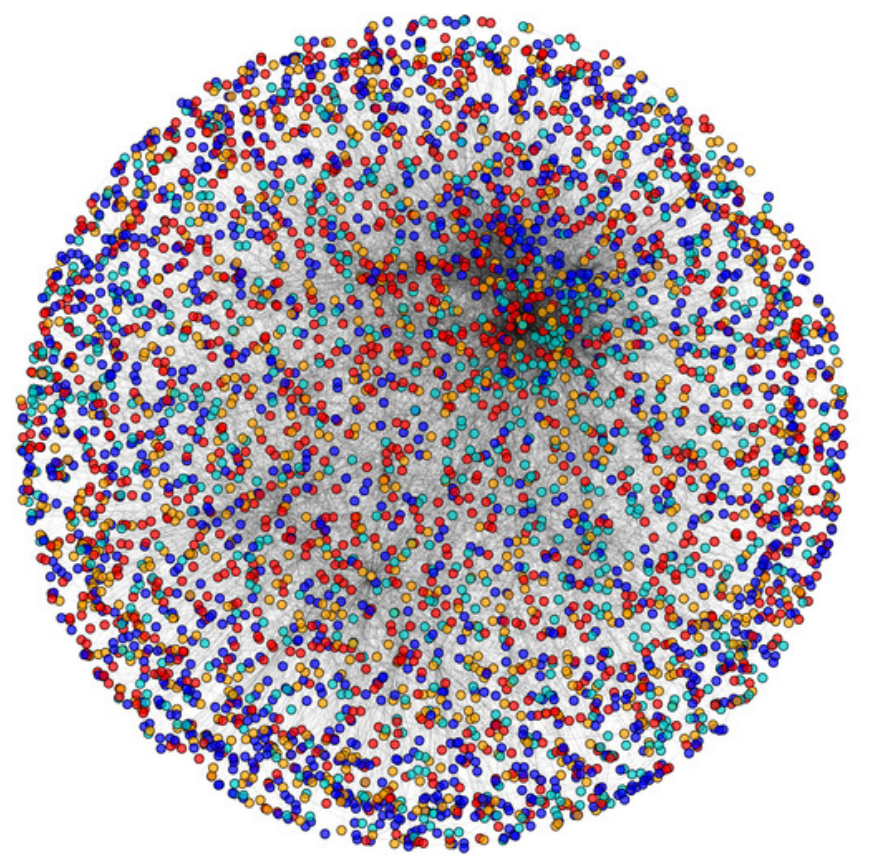


Figure 5

Sequential formation of Cytoplasm proteins (red), Nuclear Proteins (Green), Interface Proteins (Blue), Unpredicted localization Proteins (Orange) in PPIN of yeast

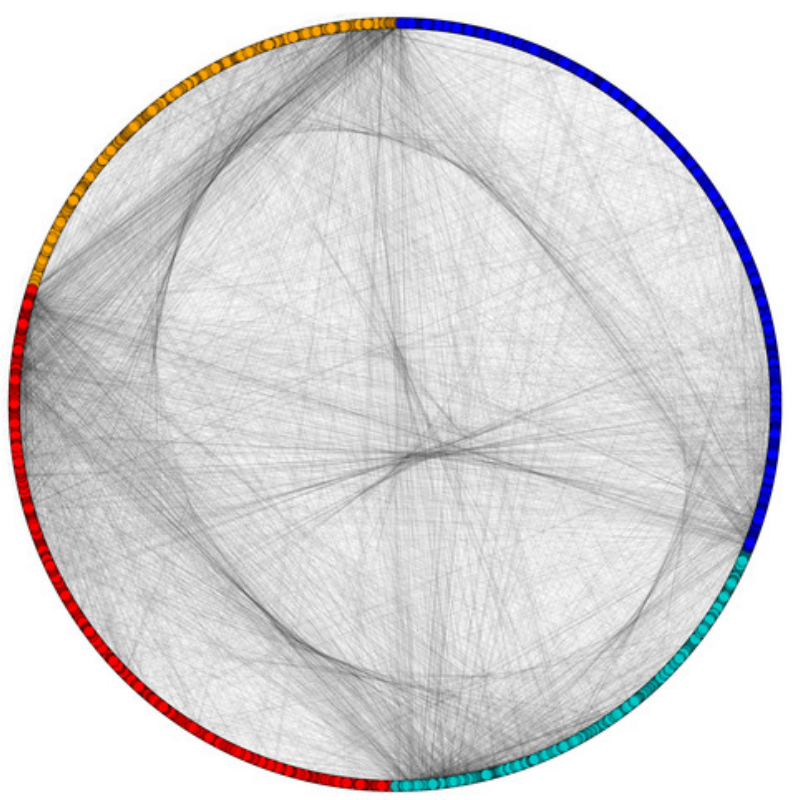


Figure 6

Separate PPIN's of Cytoplasm proteins (red), Nuclear Proteins (Green), Interface Proteins (Blue), Unpredicted localization Proteins (Orange) and their interactions

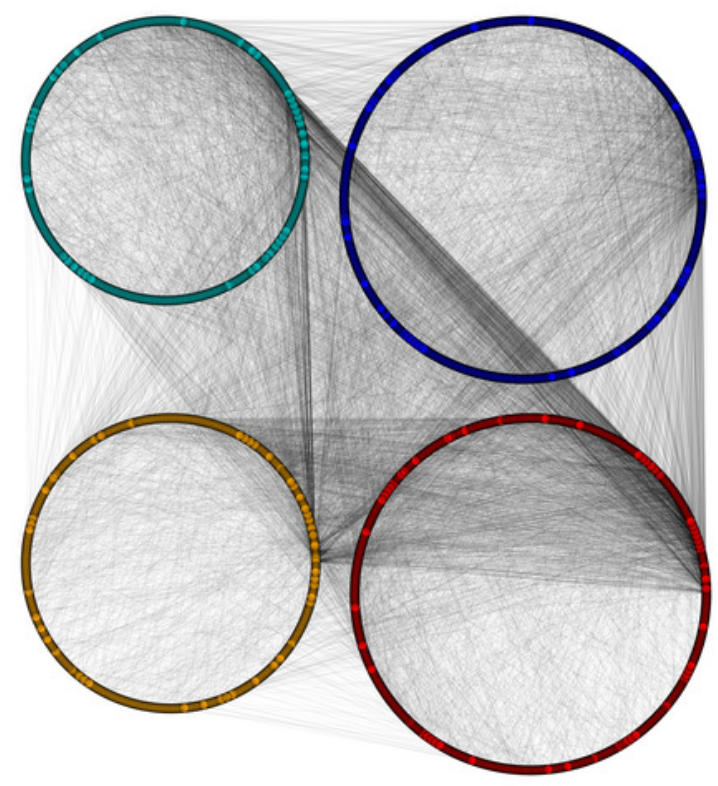




\section{Figure 7}

Candidate (green) and test (yellow) proteins in Nuclear PPIN (green and yellow) of Yeast (violet: Other nodes in the network)

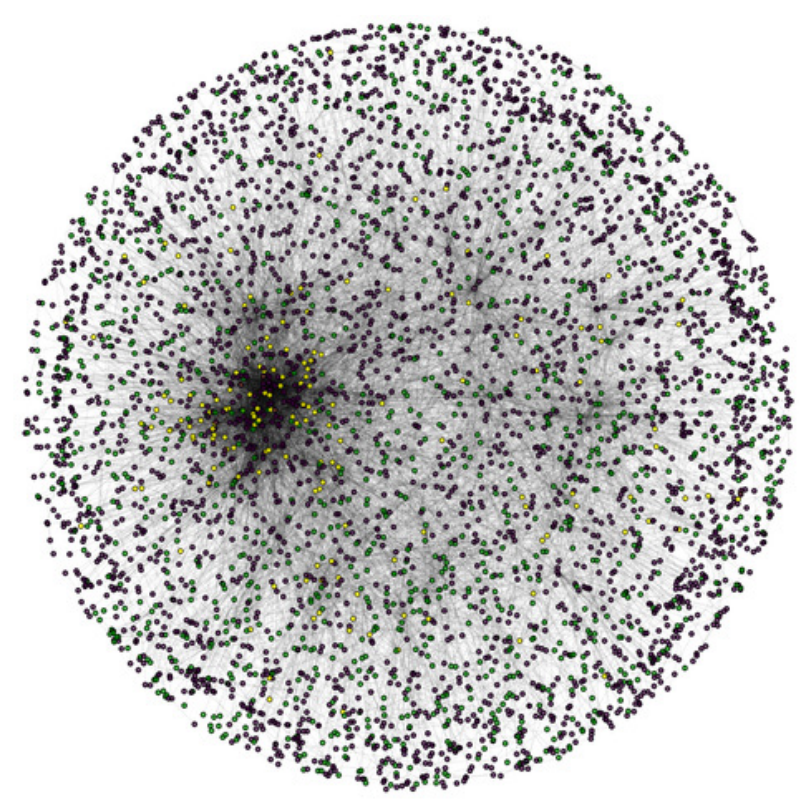




\section{Figure 8}

Candidate (red) and test (yellow) proteins in Cytoplasm PPIN (red and yellow) of Yeast (violet: Other nodes in the network)

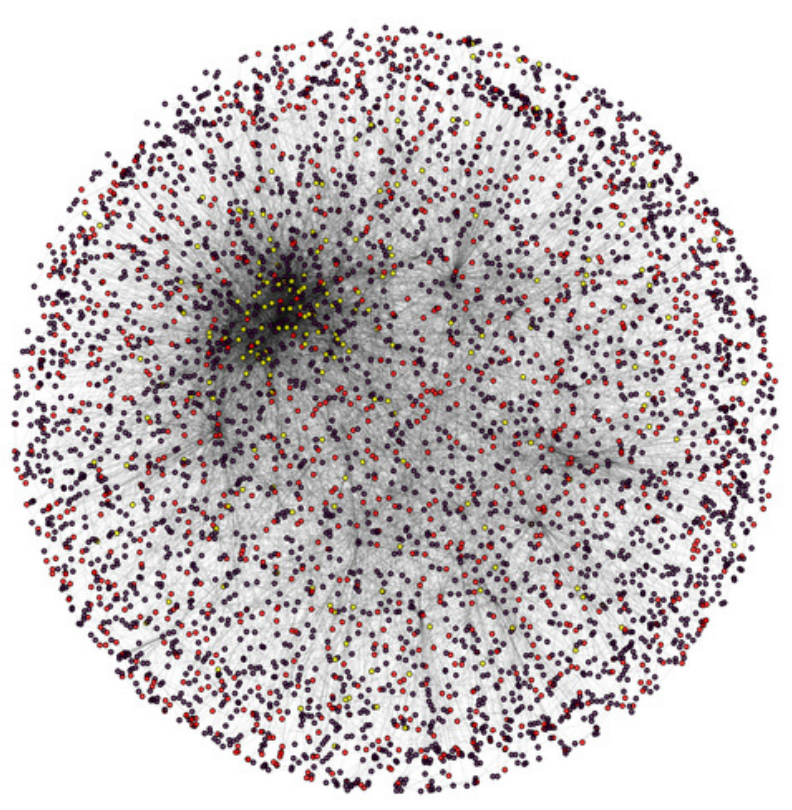




\section{Figure 9}

Candidate (blue) and test (yellow) proteins in Interface PPIN (blue and yellow) of Yeast (violet: Other nodes in the network)

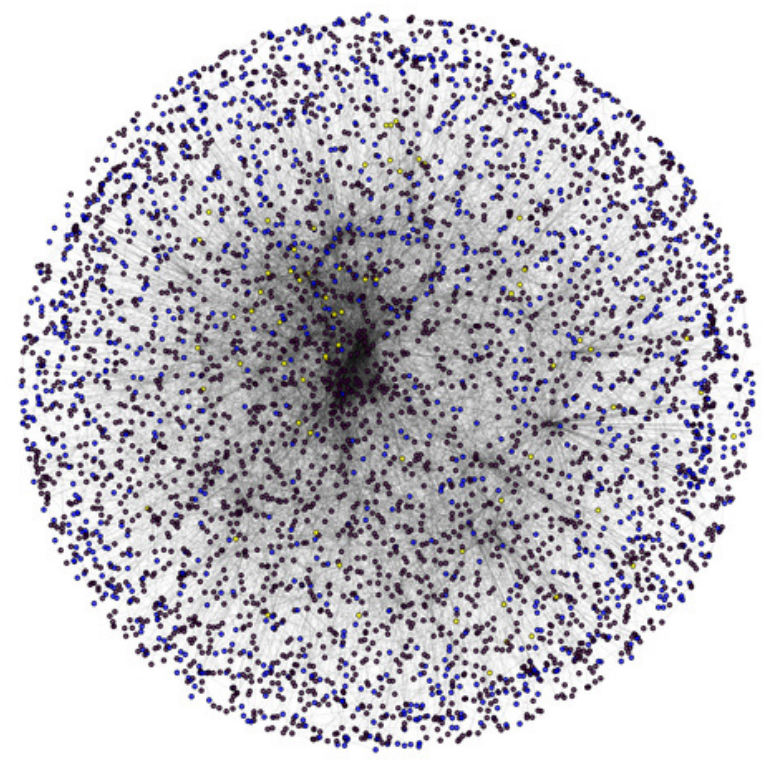


Figure 10

PPI network of Yeast (Saccharomyces cerevisiae) 


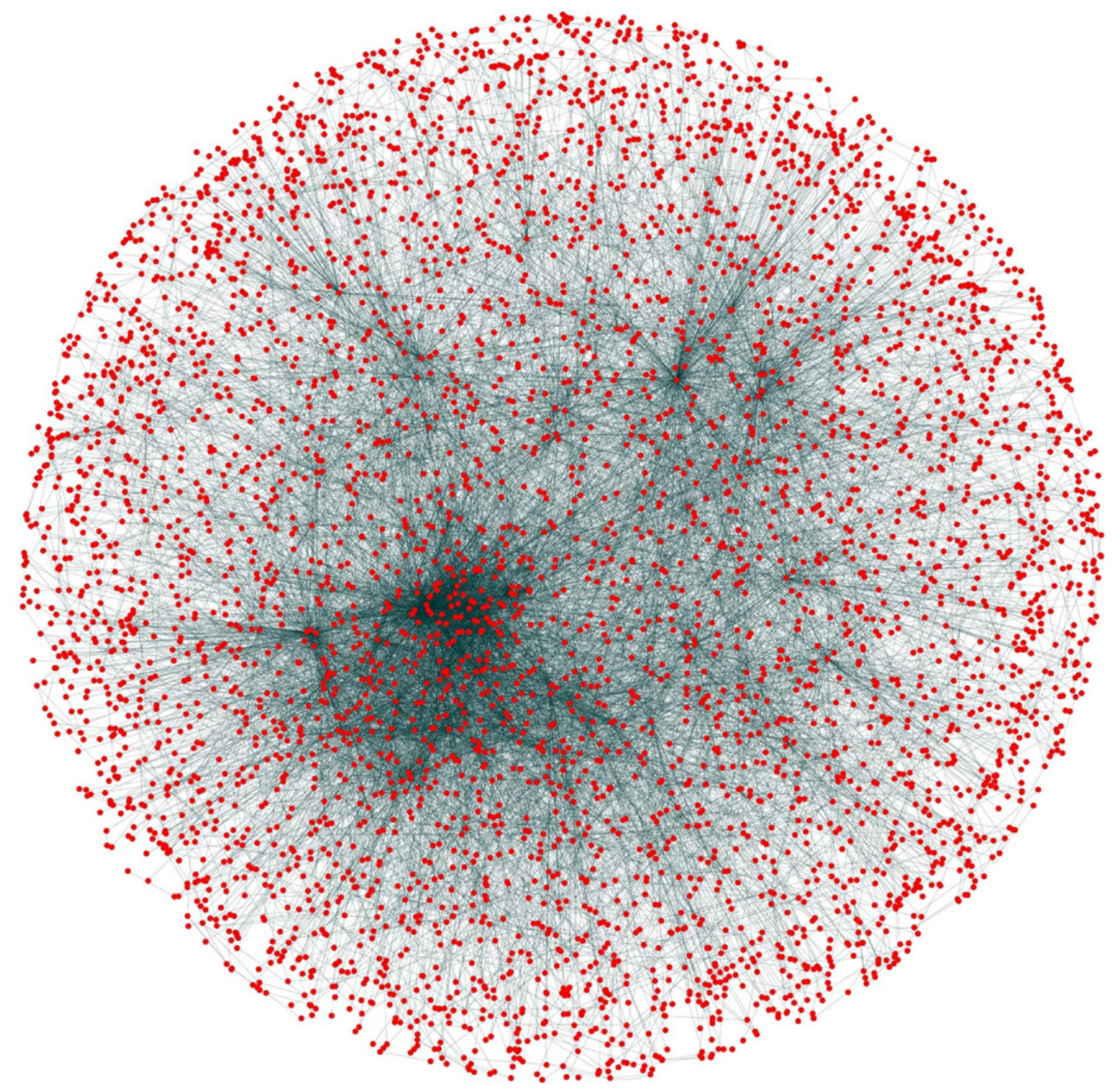


Figure 11

PPI network of annotated (red circle) and test/unannotated proteins (yellow circle) of Yeast network (Saccharomyces cerevisiae) 


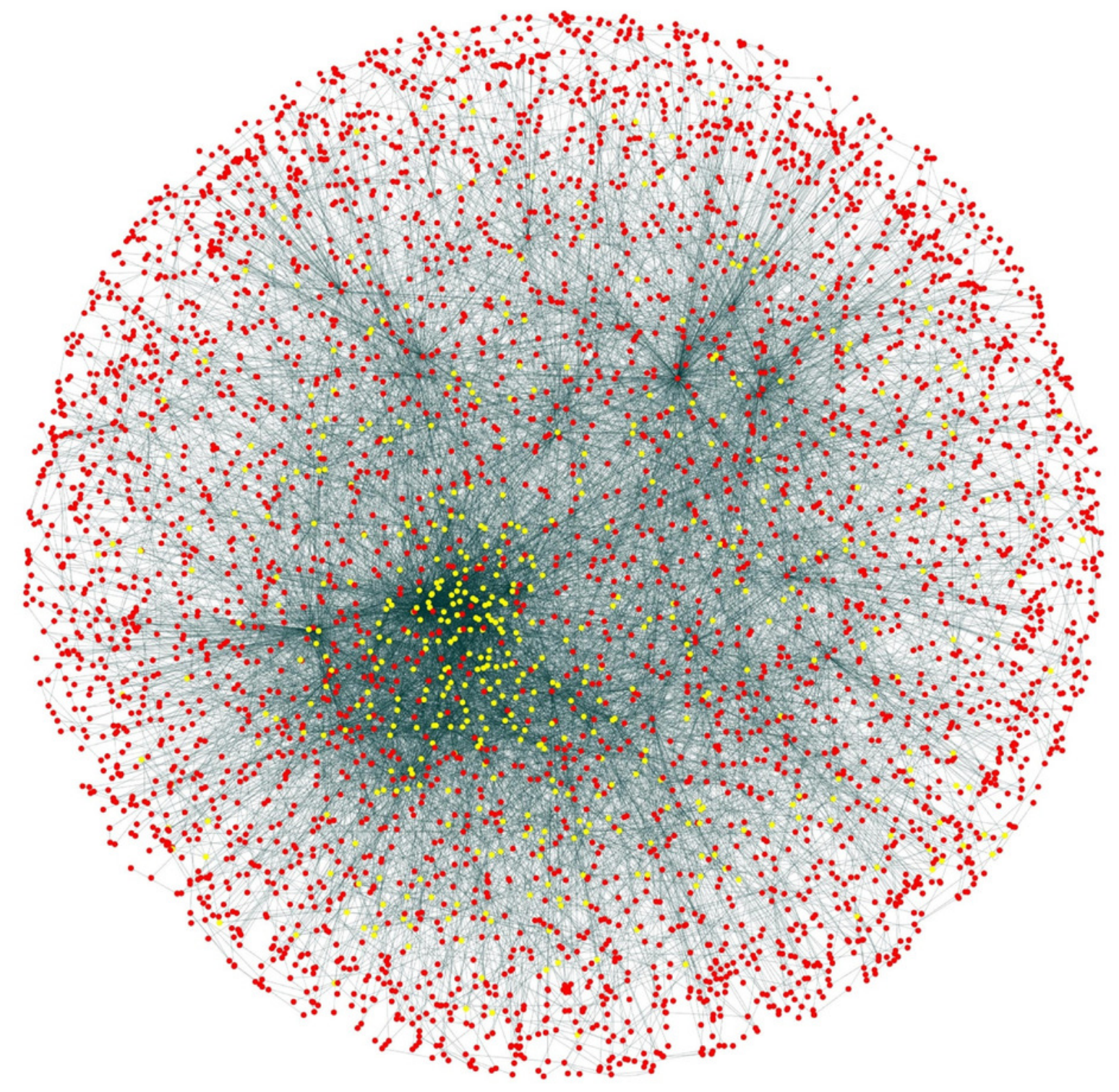




\section{Table $\mathbf{1}$ (on next page)}

Top-ranked selected physicochemical features (marked in blue) using 4 classifiers based on the maximum number of hits 


\begin{tabular}{|c|c|c|c|c|c|}
\hline \multirow[b]{2}{*}{ Physicochemical properties } & \multicolumn{5}{|c|}{$\begin{array}{c}\text { Classifiers Used } \\
\text { (Returns \#5 top-ranked physicochemical properties/features) }\end{array}$} \\
\hline & XGBoost & $\begin{array}{c}\text { Random } \\
\text { Tree }\end{array}$ & Extra Tree & $\begin{array}{l}\text { Recursive } \\
\text { Feature } \\
\text { Elimination }\end{array}$ & \#Hits \\
\hline Aromacity & $x$ & $\checkmark$ & $\checkmark$ & $x$ & 2 \\
\hline Gravy & 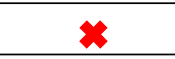 & $\checkmark$ & $\checkmark$ & $\checkmark$ & 3 \\
\hline Instability index & $x$ & $\checkmark$ & $x$ & $x$ & 1 \\
\hline Isoelectric point & $\sqrt{2}$ & $\checkmark$ & $\sqrt{2}$ & $\checkmark$ & 4 \\
\hline Negatively charged particle & $\checkmark$ & $x$ & $x$ & $\checkmark$ & 2 \\
\hline Positively charged particle & $\checkmark$ & $x$ & $\checkmark$ & $\checkmark$ & 3 \\
\hline Extinction coefficient & $\checkmark$ & $x$ & $x$ & $x$ & 1 \\
\hline Aliphatic index & $\checkmark$ & $\checkmark$ & $\checkmark$ & $x$ & 3 \\
\hline Absorbance & $x$ & $x$ & $*$ & $\checkmark$ & 1 \\
\hline Ip/mol weight & $x$ & $x$ & 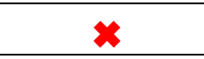 & $*$ & 0 \\
\hline
\end{tabular}


Table 2 (on next page)

Performance analyses of FunPred 3.0_Pred_SL 


\begin{tabular}{|c|c|c|c|c|c|c|}
\hline $\begin{array}{c}\text { Types of Proteins } \\
\text { (based on Subcellular- } \\
\text { localization) }\end{array}$ & $\begin{array}{c}\text { Total no. of } \\
\text { proteins in } \\
\text { database }\end{array}$ & $\begin{array}{c}\text { Total number of } \\
\text { selected annotated } \\
\text { proteins }\end{array}$ & $\begin{array}{c}\text { Total number of } \\
\text { selected essential } \\
\text { test proteins }\end{array}$ & $\begin{array}{c}\text { Prediction Accuracy } \\
\text { (Total no. of matched } \\
\text { proteins) }\end{array}$ & $\begin{array}{c}\text { Prediction } \\
\text { Accuracy (Total } \\
\text { no. of Unmatched } \\
\text { proteins) }\end{array}$ & $\begin{array}{c}\text { Failed to } \\
\text { predict }\end{array}$ \\
\hline Nuclear Proteins & 1771 & 1609 & 162 & 112 & 32 & 18 \\
\hline Cytoplasm Proteins & 1757 & 1566 & 191 & 109 & 51 & 31 \\
\hline Interface Proteins & 2246 & 2176 & 70 & 37 & 23 \\
\hline
\end{tabular}




\section{Table 3 (on next page)}

Precision, Recall and F-score obtained at three levels of node and edge weight threshold 


\begin{tabular}{|c|c|c|c|c|c|c|}
\hline $\begin{array}{c}\text { Threshold } \\
\text { Type }\end{array}$ & $\begin{array}{c}\text { Node Weight } \\
\text { Threshold }\end{array}$ & $\begin{array}{c}\text { Edge } \\
\text { Weight } \\
\text { Threshold }\end{array}$ & $\begin{array}{c}\text { Selected Test } \\
\text { Proteins }\end{array}$ & Precision & Recall & F-score \\
\hline High & 1.072 & 0.110 & 433 & 0.55 & 0.82 & 0.66 \\
\hline Medium & 1.068 & 0.109 & 433 & 0.55 & 0.82 & 0.66 \\
\hline Low & 1.064 & 0.107 & 520 & 0.54 & 0.82 & 0.65 \\
\hline
\end{tabular}




\section{Table 4(on next page)}

Performance analyses of FunPred 3.0 with other protein function prediction methodologies 


\begin{tabular}{|c|c|c|c|}
\hline Methods & Precision & Recall & F-score \\
\hline FunPred 3.0 & 0.55 & 0.82 & 0.66 \\
\hline FunPred-2 (Saha et al. 2017a) & 0.51 & 0.90 & 0.65 \\
\hline FPred_Apriori (Prasad et al., 2017) & 0.64 & 0.66 & 0.65 \\
\hline FunPred 1.1 (S. Saha et al., 2014) & 0.61 & 0.50 & 0.55 \\
\hline FunPred 1.2 (S. Saha et al., 2014) & 0.63 & 0.56 & 0.59 \\
\hline Deep_GO (Kulmanov et al. 2018) & 0.48 & 0.49 & 0.48 \\
\hline Chi square \#1\&2 (Hishigaki et al., 2001) & 0.20 & 0.25 & 0.22 \\
\hline Chi square \#1 (Hishigaki et al., 2001) & 0.25 & 0.27 & 0.26 \\
\hline Neighborhood counting \#1\&2 (Schwikowski et al., 2000) & 0.28 & 0.41 & 0.33 \\
\hline Neighborhood counting \#1 (Schwikowski et al., 2000) & 0.26 & 0.45 & 0.33 \\
\hline Fs-weight \#1\&2 (Chua et al., 2006) & 0.36 & 0.43 & 0.39 \\
\hline Fs-weight \#1 (Chua et al., 2006) & 0.33 & 0.42 & 0.37 \\
\hline Nrc (Moosavi et al., 2013) & 0.37 & 0.43 & 0.40 \\
\hline Zhang (S. Zhang et al., 2009) & 0.20 & 0.19 & 0.19 \\
\hline DCS (Peng et al., 2014) & 0.36 & 0.37 & 0.36 \\
\hline DSCP (Peng et al., 2014) & 0.39 & 0.40 & 0.39 \\
\hline PON (Liang et al., 2013) & 0.15 & 0.14 & 0.14 \\
\hline
\end{tabular}




\section{Table 5 (on next page)}

Predicted samples of Unpredicted protein pair Interactions/functions ("Missing" proteinpair-Interactions/functions) in the MIPS dataset 


\begin{tabular}{|c|c|c|c|c|c|}
\hline \multicolumn{2}{|c|}{ Interacting Protein Pairs } & \multicolumn{2}{|c|}{ Predicted Interactions } & \multicolumn{2}{c|}{ Predicted Functions } \\
\hline Protein\#1 & Protein\#2 & Interaction\#1 & Interaction\#2 & Function\#1 & Function\#2 \\
\hline YAL014c & YAL030w & two hybrid & coimmunoprecipitation & -- & -- \\
\hline YAL014c & YMR197c & two hybrid & coimmunoprecipitation & -- & -- \\
\hline YLR459w & YDR434w & Unable to Predict & -- & -- & -- \\
\hline YDR167w & YBR081c & two hybrid & -- & -- \\
\hline YGL173c & YML085c & synthetic lethal & -- & -- \\
\hline YGL190c & YKL048c & synthetic lethal & two hybrid & Cell polarity & -- \\
\hline YMR167w & YNL082w & coimmunoprecipitation & copurification & DNA repair & - \\
\hline YDR027c & YJR060w & $\begin{array}{c}\text { affinity } \\
\text { affinity-tag GST }\end{array}$ & two hybrid & -- & - \\
\hline YGR082w & YNL131w & crosslinking & coimmunoprecipitation & -- & - \\
\hline YJR066w & YHR186c & synthetic lethal & -- & -- & Lipid metabolism \\
\hline YDR363w-a & YER008c & synthetic lethal & -- & Vesicular transport & -- \\
\hline YDR309c & YLR319c & synthetic lethal & Cell structure & -- & -- \\
\hline YLR336c & YPL268w & Unable to Predict & -- & -- & - \\
\hline YKR099w & YDL106c & Unable to Predict & -- & - \\
\hline
\end{tabular}


Table 6 (on next page)

Predicted samples of Unpredicted protein pair Interactions/functions ("Unknown" protein-pair-Interactions/functions) in the MIPS dataset 


\begin{tabular}{|c|c|c|c|c|c|}
\hline \multicolumn{2}{|c|}{ Interacting Protein Pairs } & \multicolumn{2}{|c|}{ Predicted Interactions } & \multicolumn{2}{|c|}{ Predicted Functions } \\
\hline Protein\#1 & Protein\#2 & Interaction\#1 & Interaction $\# 2$ & Function\#1 & Function\#2 \\
\hline YLR418c & YIL040w & two hybrid & -- & Pol II Transcription & -- \\
\hline YOR326w & YNL120c & Mitosis & -- & Cell polarity & $\begin{array}{c}\text { Cell cycle } \\
\text { control }\end{array}$ \\
\hline YJR057w & YDR438w & Unable to Predict & -- & -- & -- \\
\hline YFL037w & YMR299c & Cell structure & -- & RNA processing & DNA repair \\
\hline YHR129c & YGL124c & Mitosis & two hybrid & -- & -- \\
\hline YGR078c & YAL011w & synthetic lethal & two hybrid & -- & -- \\
\hline YNL153c & YDR149c & two hybrid & -- & Pol II transcription & -- \\
\hline YMR307w & YMR317w & two hybrid & -- & $\begin{array}{c}\text { Carbohydrate } \\
\text { metabolism }\end{array}$ & -- \\
\hline YLR039c & YIL039w & Vesicular transport & two hybrid & -- & -- \\
\hline YMR307w & YHR004c & two hybrid & -- & $\begin{array}{l}\text { Carbohydrate } \\
\text { metabolism }\end{array}$ & -- \\
\hline YDL003w & YGL250w & two hybrid & -- & Energy generation & -- \\
\hline YNL271c & YGR228w & Meiosis & -- & Cell polarity & $\begin{array}{c}\text { Protein } \\
\text { modification }\end{array}$ \\
\hline YML094w & YBR108w & Unable to Predict & -- & -- & -- \\
\hline YEL003w & YDR334w & Unable to Predict & -- & -- & -- \\
\hline
\end{tabular}

